\title{
Agricultural and Related Pest Control Applicator License Classifications under the Florida Department of Agriculture and Consumer Services (FDACS) ${ }^{1}$
}

\section{Frederick M. Fishel ${ }^{2}$}

This guide provides an explanation of private, public and commercial pesticide applicator licenses issued by FDACS under Chapter 487 Florida Statutes.

\section{Private Applicator}

A private applicator is a licensed applicator who applies restricted use pesticides by ground application for the purpose of producing an agricultural commodity on property owned or rented by the applicator or the applicator's employer.

The license is appropriate for owners and employees of farms, ranches, groves, nurseries, gardens, and other establishments that produce agricultural commodities. The license is valid for application of restricted use pesticides for ornamental and turf production (at a nursery, sod farm, etc.), but not for maintenance of ornamentals or turf, such as at a golf course.

The private applicator agricultural pest control category is required for this license type. Effective in September 2004, the aerial category is not available to private applicators.
A commercial applicator license may substitute for a private applicator license for certain uses. For further discussion, see the section on commercial applicators.

\section{Public Applicator}

A public applicator is a licensed applicator employed by a federal, state, county, city, or other local agency or government entity who uses restricted use pesticides on the job. This license is valid for work performed for the government agency during the course of employment. The following must have a public applicator license:

- Employees of municipal electric companies, city and county parks, government-owned railways, state and federal highway departments, and state universities who use restricted use pesticides on the job;

- Extension agents, public university researchers, and vocational agriculture instructors who use or demonstrate the use of restricted use pesticides must have a public applicator license including the demonstration and research category; and,

1. This document is PI-59, one of a series of the Pesticide Information Office, Florida Cooperative Extension Service, Institute of Food and Agricultural Sciences, University of Florida. Original publication date August 2005. Visit the EDIS Web Site at http://edis.ifas.ufl.edu.

2. Frederick M. Fishel, Associate Professor, Agronomy Department, and Director, Pesticide Information Office; Florida Cooperative Extension Service, Institute of Food and Agricultural Sciences, University of Florida, Gainesville, FL 32611.

The Institute of Food and Agricultural Sciences (IFAS) is an Equal Opportunity Institution authorized to provide research, educational information and other services only to individuals and institutions that function with non-discrimination with respect to race, creed, color, religion, age, disability, sex, sexual orientation, marital status, national origin, political opinions or affiliations. U.S. Department of Agriculture, Cooperative Extension Service, University of Florida, IFAS, Florida A. \& M. University Cooperative Extension Program, and Boards of County Commissioners Cooperating. Larry Arrington, Dean 
- Federal and state personnel conducting pest eradication or control using restricted use pesticides must have a public applicator license including the regulatory pest control category.

NOTE: A commercial applicator license may substitute for a public applicator license if desired. However, liability issues should be taken into consideration and discussed with the employer if an applicator plans to use a commercial license for making pesticide applications as part of employment with a government entity.

\section{Commercial Applicator}

A commercial applicator is a person who uses restricted use pesticides in any agricultural or related area for any purpose other than as provided by the other license classifications. This includes the following uses:

- Contract application of restricted use pesticides for someone else (when someone other than the owner or an employee of the firm makes the application).

- Non-contract application of restricted use pesticides for any purpose other than agricultural production, but not including work-related applications made by government employees.

Examples of the licenses required for many types of work being conducted are provided in Table 1. Some of the common situations that occur in Florida where a commercial license is required include:

- Aerial application service (not including public health aerial application)

- Aquatic weed control service

- Cemetery maintenance service

- Park maintenance - recreational (privately owned), including athletic fields

- Golf course - turf or greens maintenance

- Chemical/fertilizer company - custom application
- Contract right-of-way pest control for government agencies or utilities

- Seed treatment company

- Field or greenhouse research and demonstration by industry representatives

- Field or greenhouse research and demonstration by private researchers

An applicator may make applications of restricted use pesticides only in the categories for which he has been licensed. For example, a golf course applicator must be licensed in the ornamental and turf category to apply restricted use pesticides to the golf course. If he applies a restricted use pesticide to a pond on the golf course, he must also be licensed in the aquatic category.

NOTE: A commercial applicator license may substitute for a private applicator license only for uses covered by the commercial license. For example, a commercial license with the agricultural row crop category only may substitute for applications made to row crops on property owned or rented by the applicator or the applicator's employer (but would not be valid for application to tree crops, ornamentals or turf, forest sites, aquatic sites, or for animal treatment, seed treatment, wood treatment, or fumigation). 
Agricultural and Related Pest Control Applicator License Classifications under the....

Table 1. Certification and license requirements based on pesticide use.

\begin{tabular}{|c|c|c|c|}
\hline \multirow[t]{2}{*}{ Pesticide use } & \multicolumn{3}{|c|}{ Certification or License required } \\
\hline & Private & Public & Commercial \\
\hline \multicolumn{4}{|l|}{$\begin{array}{l}\text { AERIAL (agricultural production including ag, forestry, turf, } \\
\text { etc.) }\end{array}$} \\
\hline By property owner or non-government employee & & & $\mathrm{x}$ \\
\hline By government employee (work related) & & $\mathrm{X}$ & $\mathrm{X}$ \\
\hline By contract service & & & $\mathrm{x}$ \\
\hline \multicolumn{4}{|l|}{ AQUATIC } \\
\hline By property owner or employee (agricultural production only) & $\mathrm{X}$ & & \\
\hline $\begin{array}{l}\text { By property owner or employee (anything other than } \\
\text { agricultural production) }\end{array}$ & & & $\mathrm{x}$ \\
\hline $\begin{array}{l}\text { By government employee (agricultural or anything other } \\
\text { than public health) }\end{array}$ & & $\mathrm{x}$ & \\
\hline \multicolumn{4}{|l|}{ CHEMICAL/FERTILIZER COMPANY } \\
\hline By employees who demonstrate or custom blend RUPs & & & $\mathrm{X}$ \\
\hline \multicolumn{4}{|l|}{ FARM, GROVE, RANCH } \\
\hline By property owner or non-government employee & $\mathrm{X}$ & & $\mathrm{x}$ \\
\hline By government employee (work related) & & $\mathrm{x}$ & $\mathrm{x}$ \\
\hline By contract service & & & $\mathrm{x}$ \\
\hline \multicolumn{4}{|l|}{ FERNERY (fern nursery) } \\
\hline By property owner or non-government employee & $x$ & & $\mathrm{X}$ \\
\hline By government employee (work related) & & $\mathrm{x}$ & $\mathrm{X}$ \\
\hline By contract service & & & $\mathrm{x}$ \\
\hline \multicolumn{4}{|l|}{ FUMIGATION (soil or greenhouse) } \\
\hline By property owner or non-government employee & $x$ & & $\mathrm{X}$ \\
\hline By government employee (work related) & & $\mathrm{x}$ & $\mathrm{X}$ \\
\hline By contract service & & & $\mathrm{x}$ \\
\hline \multicolumn{4}{|l|}{ FUMIGATION (raw harvested agricultural commodities } \\
\hline By property owner or non-government employee & $\mathrm{X}$ & & $\mathrm{X}$ \\
\hline By government employee (work related) & & $\mathrm{x}$ & $\mathrm{x}$ \\
\hline By contract service & & & $\mathrm{x}$ \\
\hline \multicolumn{4}{|l|}{ GARDEN (home garden) } \\
\hline By property owner or non-government employee & $\mathrm{x}$ & & $\mathrm{x}$ \\
\hline By government employee (work related demonstration, etc.) & & $\mathrm{x}$ & $\mathrm{x}$ \\
\hline By contract service & & & $\mathrm{X}$ \\
\hline \multicolumn{4}{|l|}{ GOLF COURSE, PARK, CEMETERY } \\
\hline By property owner or non-government employee & & & $\mathrm{x}$ \\
\hline By government employee (work related) & & $\mathrm{x}$ & $\mathrm{x}$ \\
\hline By contract service & & & $\mathrm{x}$ \\
\hline \multicolumn{4}{|l|}{ GREENHOUSE } \\
\hline By property owner on non-government employee & $\mathrm{x}$ & & $\mathrm{x}$ \\
\hline By government employee (work related) & & $\mathrm{X}$ & $\mathrm{x}$ \\
\hline By contract service & & & $\mathrm{x}$ \\
\hline \multicolumn{4}{|l|}{ INTERIORSCAPING \& INDOOR PLANT MAINTENANCE } \\
\hline $\begin{array}{l}\text { By employee of a nursery that provides plants to facilities but } \\
\text { removes the plants from the facility and takes them back to } \\
\text { the nursery or greenhouse for pest control }\end{array}$ & & & $\mathrm{x}$ \\
\hline
\end{tabular}


Agricultural and Related Pest Control Applicator License Classifications under the....

Table 1. Certification and license requirements based on pesticide use.

\begin{tabular}{|c|c|c|c|}
\hline \multirow[t]{2}{*}{ Pesticide use } & \multicolumn{3}{|c|}{ Certification or License required } \\
\hline & Private & Public & Commercial \\
\hline \multicolumn{4}{|l|}{ NURSERY OR SOD FARM } \\
\hline By owner or non-government employee of nursery, etc. & $\mathrm{x}$ & & \\
\hline By government employee (forest seed bed, etc.) & & $x$ & \\
\hline By contract service & & & $\mathrm{x}$ \\
\hline \multicolumn{4}{|l|}{ RESEARCH (FIELD PLOTS) } \\
\hline By government employee & & $\mathrm{x}$ & \\
\hline $\begin{array}{l}\text { Any person other than government employee including } \\
\text { private industry and contract service }\end{array}$ & & & $\mathrm{x}$ \\
\hline \multicolumn{4}{|l|}{ RIGHT-OF-WAY } \\
\hline By government employee & & $x$ & \\
\hline By anyone else, including contract service & & & $\mathrm{x}$ \\
\hline \multicolumn{4}{|l|}{ SEED TREATMENT } \\
\hline By government employee & & $\mathrm{x}$ & \\
\hline \multicolumn{4}{|l|}{ By anyone else: } \\
\hline $\begin{array}{l}\text { 1. Seed to be used on licensee's property in ag } \\
\text { production }\end{array}$ & $\mathrm{x}$ & & \\
\hline $\begin{array}{l}\text { 2. Seed to be sold or used for any purpose other } \\
\text { than ag production on licensee's property }\end{array}$ & & & $\mathrm{x}$ \\
\hline \multicolumn{4}{|l|}{ SEWER ROOT CONTROL } \\
\hline By government employee & & $x$ & \\
\hline By anyone else & & & $\mathrm{X}$ \\
\hline \multicolumn{4}{|l|}{ WILDLIFE HABITAT MANAGEMENT } \\
\hline $\begin{array}{l}\text { By owner or employee of wildlife habitat area (plantation, } \\
\text { etc.) }\end{array}$ & & & $x$ \\
\hline By government employee & & $\mathrm{x}$ & \\
\hline By contract service to one of the above & & & $x$ \\
\hline \multicolumn{4}{|l|}{ WOOD TREATMENT } \\
\hline By government employee & & $\mathrm{X}$ & \\
\hline \multicolumn{4}{|l|}{ By anyone else: } \\
\hline $\begin{array}{l}\text { 1. Wood to be used on licensee's property for ag } \\
\text { production }\end{array}$ & $x$ & & \\
\hline $\begin{array}{l}\text { 2. Wood to be sold or used for any purpose other } \\
\text { than agricultural production on licensee's property }\end{array}$ & & & $x$ \\
\hline
\end{tabular}

